\title{
Simultaneous Mapping of Pan and Sentinel Lymph Nodes for Real-Time Image-Guided Surgery
}

\author{
Yoshitomo Ashitate ${ }^{1,2^{*}}$, Hoon Hyun ${ }^{1 *}$, Soon Hee Kim ${ }^{1}$, Jeong Heon Lee ${ }^{1}$, Maged Henary ${ }^{3}$, John V. \\ Frangioni $^{1,4}$, Hak Soo Choi ${ }^{1,5 凶}$ \\ 1. Division of Hematology/Oncology, Department of Medicine, Beth Israel Deaconess Medical Center, Boston, MA 02215, USA \\ 2. Division of Cancer Diagnostics and Therapeutics, Hokkaido University Graduate School of Medicine, Sapporo 060-8638, Japan \\ 3. Department of Chemistry, Georgia State University, Atlanta, GA 30303, USA \\ 4. Department of Radiology, Beth Israel Deaconess Medical Center, Boston, MA 02215, USA \\ 5. Department of Cogno-Mechatronics Engineering, Pusan National University, Busan 609-735, South Korea \\ * These authors contributed equally to this work. \\ $\square$ Corresponding author: Hak Soo Choi, Ph.D. 330 Brookline Avenue, Room SL-436A, Boston, MA 02215. Tel: 617-667-6024, Fax: \\ 617-975-5016; E-mail: hchoi@bidmc.harvard.edu.
}

(c) Ivyspring International Publisher. This is an open-access article distributed under the terms of the Creative Commons License (http://creativecommons.org/ licenses/by-nc-nd/3.0/). Reproduction is permitted for personal, noncommercial use, provided that the article is in whole, unmodified, and properly cited.

Received: 20I4.0I.30; Accepted: 2014.03.24; Published: 20I4.04.24

\begin{abstract}
The resection of regional lymph nodes in the basin of a primary tumor is of paramount importance in surgical oncology. Although sentinel lymph node mapping is now the standard of care in breast cancer and melanoma, over $20 \%$ of patients require a completion lymphadenectomy. Yet, there is currently no technology available that can image all lymph nodes in the body in real time, or assess both the sentinel node and all nodes simultaneously. In this study, we report an optical fluorescence technology that is capable of simultaneous mapping of pan lymph nodes (PLNs) and sentinel lymph nodes (SLNs) in the same subject. We developed near-infrared fluorophores, which have fluorescence emission maxima either at $700 \mathrm{~nm}$ or at $800 \mathrm{~nm}$. One was injected intravenously for identification of all regional lymph nodes in a basin, and the other was injected locally for identification of the SLN. Using the dual-channel FLARE intraoperative imaging system, we could identify and resect all PLNs and SLNs simultaneously. The technology we describe enables simultaneous, real-time visualization of both PLNs and SLNs in the same subject.
\end{abstract}

Key words: Near-infrared fluorescence, pan lymph node mapping, completion lymphadenectomy, sentinel lymph node mapping, image-guided surgery, cervical cancer.

\section{Introduction}

The sentinel lymph node (SLN) is the first lymph node (LN) receiving lymphatic drainage from a primary tumor [1]. SLN biopsy is essential for staging many cancers and avoiding unnecessary LN dissection, which may cause adverse effects such as lymphedema. Currently, SLN biopsy is the accepted standard of care for breast cancer and melanoma [2]. Although SLN biopsy studies have been attempted for colorectal cancer, gastric cancer, esophageal cancer, head and neck cancer, thyroid cancer, non-small cell lung cancer, and gynecologic cancer, the importance of SLN biopsy for these cancers remains unclear [3-9].
Regardless of SLN status, the prognosis of almost all solid tumors is dependent on LN involvement, and even in the case of a positive SLN biopsy, completion lymphadenectomy is required in $15-40 \%$ of cases [10-13]. Despite these facts, there is currently no technology available to the surgeon to identify all LNs within a basin quickly and with high sensitivity. Previous reports demonstrated that the use of invisible near-infrared (NIR) light (700 - $900 \mathrm{~nm}$ ) has several advantages for SLN biopsy, including relatively high penetration (up to 5-8 $\mathrm{mm}$ ) into living tissue [14, 15]. When compared to blue dyes, invisible NIR light has a relatively high signal-to-background ratio (SBR) due 
to low absorption, scattering, and tissue autofluorescence [16]. NIR light is also non-ionizing. Because of these advantages, many recent studies have used NIR fluorescence for SLN biopsy [17-24], reviewed in [25]. PLN mapping, however, has not yet been widely investigated. Josephson et al. employed Cy5.5-grafted polymers (PGC) to demonstrate NIR imaging of PLNs; however, nonspecific background was so high in major organs including liver, spleen, and lungs that the entire viscera had to be removed to identify the LNs after intravenous injection [26].

In this study, we engineered novel small molecule-based polymethine cyanine fluorophores to have fluorescence emission maxima in two distinct ranges; one NIR color for visualization of PLNs and the other for mapping SLN in the same subject. These LN-targeted NIR fluorophores were injected into small and large animal models, and their targeting efficiency in PLN and SLN was evaluated using the dual-channel FLARE intraoperative imaging system in real time.

\section{Materials and Methods}

Optical Property Measurements. We synthesized a series of endocrine-specific near-infrared fluorophores (ESNF), and used them for lymph node targeting with a previously reported zwitterionic (ZW) heptamethine indocyanine molecule ZW800-3a [27]. To compare current commercially available NIR fluorophores, methylene blue (MB) purchased from Taylor Pharmaceuticals (Decatur, IL) and indocyanine green (ICG) purchased from Akorn Inc. (Decatur, IL) were also prepared. The optical properties of all agents were measured in fetal bovine serum (FBS) supplemented with $50 \mathrm{mM}$ HEPES, $\mathrm{pH}$ 7.4. For quantum yield (QY) measurements of $700 \mathrm{~nm}$ fluorophores (ESNF11, -14, -20), oxazine 725 in ethylene glycol (QY $=19 \%)$ was used as a calibration standard under conditions of matched absorbance at $655 \mathrm{~nm}$ $[28,29]$. For the QY of $800 \mathrm{~nm}$ fluorophores (ZW800-3a), ICG in DMSO (QY $=13 \%$ ) was used with a matched absorbance at $770 \mathrm{~nm}[30,31]$. For in vitro optical property measurements, fiberoptic HR2000 absorbance (200-1100 nm) and USB2000FL fluorescence (350-1000 nm) spectrometers (Ocean Optics, Dunedin, FL) were employed. NIR excitation was provided by a $770 \mathrm{~nm}$ NIR laser diode light source (Electro Optical Components, Santa Rosa, CA) set to 8 $\mathrm{mW}$ and coupled through a $300 \mu \mathrm{M}$ core diameter, 0.22 NA fiber (Fiberguide Industries, Stirling, NJ). In silico calculations of the partition coefficient (log D) and surface molecular charge and hydrophobicity were calculated using MarvinSketch 5.2.1 (ChemAxon, Budapest, Hungary) by evaluating major microspecies at $\mathrm{pH} 7.4$.
Animal Models. Animals were housed in an AAALAC-certified facility. Animal studies were performed under the supervision of Beth Israel Deaconess Medical Center's Institutional Animal Care and Use Committee (IACUC) in accordance with approved institutional protocols \#155-2008 and \#046-2010. We anesthetized male $300 \mathrm{~g}$ Sprague-Dawley (SD) rats (Taconic Farms, Germantown, $\mathrm{NY)}$ with $80 \mathrm{mg} / \mathrm{kg}$ ketamine and $8 \mathrm{mg} / \mathrm{kg}$ xylazine intraperitoneally (Webster Veterinary, Fort Devens, MA). Female Yorkshire pigs (E.M. Parsons and Sons, Hadley, MA) averaging $35.4 \mathrm{~kg}$ were induced with 4.4 $\mathrm{mg} / \mathrm{kg}$ intramuscular Telazol ${ }^{\mathrm{TM}}$ (Fort Dodge Labs, Fort Dodge, IA), intubated, and maintained with $2 \%$ isoflurane (Baxter Healthcare Corp., Deerfield, IL). Following anesthesia, a $14 \mathrm{G}$ central venous catheter was inserted into the external jugular vein, and saline was administered as needed. Electrocardiogram, heart rate, pulse oximetry, and body temperature were monitored throughout surgery.

NIR Fluorescence Imaging System. The dual-NIR channel FLARE imaging system has been described in detail previously [20, 30, 32]. In this study, $670 \mathrm{~nm}$ excitation and $760 \mathrm{~nm}$ excitation fluorescence rates used were 4.0 and $11.0 \mathrm{~mW} / \mathrm{cm}^{2}$, respectively, with white light (400 to $650 \mathrm{~nm}$ ) at 40,000 lx. Color image and 2 independent channels $(700 \mathrm{~nm}$ and 800 $\mathrm{nm}$ ) of NIR fluorescence images were acquired simultaneously with custom software at rates up to $15 \mathrm{~Hz}$ over a $15 \mathrm{~cm}$ diameter field of view (FOV). A custom filter set (Chroma Technology Corporation, Brattleboro, VT) composed of a $750 \pm 25 \mathrm{~nm}$ excitation filter, a $785 \mathrm{~nm}$ dichroic mirror, and an $810 \pm 20 \mathrm{~nm}$ emission filter were used to detect ZW800-3a and ICG. For ESNF detection, we used $650 \pm 22 \mathrm{~nm}$ and $710 \pm 25 \mathrm{~nm}$ excitation and emission filters, respectively. In the color-NIR merged images, $700 \mathrm{~nm}$ NIR fluorescence and $800 \mathrm{~nm}$ fluorescence were pseudo-colored bright red and lime green, respectively. The imaging system was positioned at a distance of 18 inches from the surgical field.

NIR Fluorescence Microscopy. NIR fluorescence microscopy was performed on a 4-filter set Nikon Eclipse TE300 epifluorescence microscope as previously described $[33,34]$. The microscope was equipped with a $100 \mathrm{~W}$ mercury light source, NIR-compatible optics, and a NIR-compatible 10X Plan Fluor objective lens and a 100X Plan Apo oil immersion objective lens (Nikon, Melville, NY). Images were acquired on an Orca-AG (Hamamatsu, Bridgewater, NJ). Image acquisition and analysis was performed using IPLab software (Scanalytics, Fairfax, VA). The same NIR filter sets used for intraoperative FLARE imaging were used for microscopic imaging.

NIR Imaging of Lymph Nodes in Rats and Pigs. 
As Josephson et al. reported [26], intravenous injection rather than local injection should be used for PLN mapping to ensure labeling of all LNs. For PLN mapping using $700 \mathrm{~nm}$ NIR fluorophores, $50 \mathrm{nmol}$ of MB or ESNF fluorophores were injected intravenously in rats. Injections of $20 \mathrm{nmol}$ of ICG and ZW800-3a were used for $800 \mathrm{~nm}$ NIR imaging ( $\mathrm{n}=3$ animals for each fluorophore). Four hours after injection, neck, axillary, inguinal, iliac, and mediastinal LNs were imaged. As a control, 3 rats were injected with $0.5 \mathrm{~mL}$ of saline and imaged as above. NIR fluorophores showing significantly higher SBR (SBR $\geq 1.3$ ) than the control (SBR $\approx 1$ ) were employed for further study. To examine the relationship between SBR of the LNs and observation timing, we observed 7 different time points $(1,2,4,8$, 12,16 , and $24 \mathrm{~h}$ ) post-injection for each fluorophore (n $=3$ animals for each).

The same series of fluorophores were also used in a large animal study. The doses of contrast agents used for the large animals were as follows: $1.4 \mu \mathrm{mol}$ for ESNF series and $640 \mathrm{nmol}$ for ZW800-3a. First, the major LNs such as inguinal, gastric, mesenteric, and mediastinal LNs were observed $4 \mathrm{~h}$ post-injection $(\mathrm{n}=$ 3 animals for each fluorophore). Second, the NIR fluorophore showing significantly higher fluorescence intensity (FI) was selected and injected into $n=3$ pigs. LNs, muscle, liver, and kidney were observed at the indicated time (10 min, $30 \mathrm{~min}, 1 \mathrm{~h}, 2 \mathrm{~h}, 3 \mathrm{~h}$, and $4 \mathrm{~h}$ ). Finally, 3 pigs were used for dual-channel imaging of SLN and PLN mapping using ESNF and ZW800-3a simultaneously. Intravenously injected ZW800-3a (640 nmol in $0.5 \mathrm{~mL}$ saline) was used for PLN mapping, and 5 nmol ESNF injected locally into the uterine cervix was used to visualize the SLN. The local injection procedure was the same method used in a recent clinical study by our group [24].

\section{$700 \mathrm{~nm}$ NIR Fluorophores}
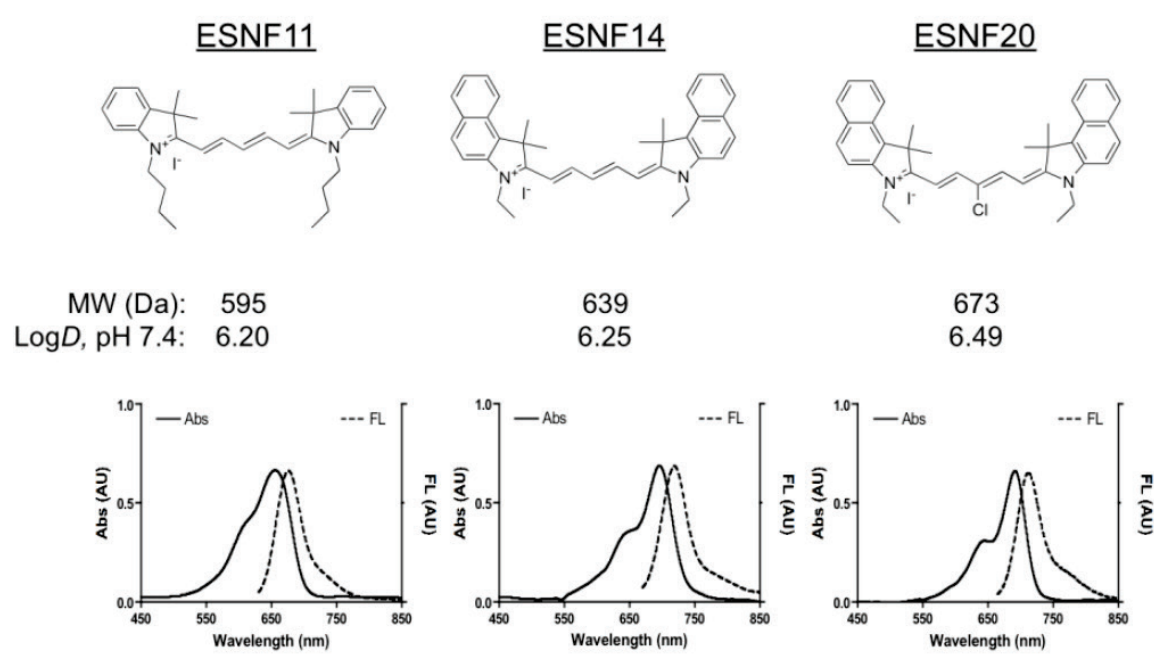

Figure I. Chemical Structures and Optical Properties of Lymph Node Contrast Agents: Shown are chemical structures (top) and optical properties (bottom) of 700 $\mathrm{nm}$ (left) and $800 \mathrm{~nm}$ (right) lymph node contrast agents. FL, fluorescence; MW, molecular weight.
Quantitation and Statistical Analysis. The FI of a region of interest (ROI) over the LN, rectus abdominis muscles, liver, and kidney were quantified using custom FLARE software. The performance metric for this study was the signal-to-background ratio (SBR). $\mathrm{SBR}=\mathrm{FI}$ of ROI / background (BG) intensity. The rectus abdominis muscles, liver, and kidney were used as BG compared to the signal in LNs to yield SBR (LN/Mu), SBR (LN/Li), and SBR (LN/Ki). Results were presented as mean \pm SEM. A one-way ANOVA followed by Tukey's multiple comparisons post-test were used to assess the statistical differences between multiple groups. A $P$ value $\leq 0.05$ was considered significant.

\section{Results}

Optical Properties of NIR Fluorophores. The chemical structure, absorbance, and fluorescence curves for the ESNF series and ZW800-3a are shown in Figure 1. Detailed optical properties in $100 \%$ serum, pH 7.4 are shown in Table 1. All ESNF fluorophores have a 2-fold higher extinction coefficient and greater than 4-fold higher QY compared to the clinically available $700 \mathrm{~nm}$ fluorophore MB. The optical properties of ZW800-3a also appear to be higher (4-fold brighter) than the clinically available $800 \mathrm{~nm}$ fluorophore ICG. ESNFs and ZW800-3a also have ideal separation in absorbance and fluorescence spectra, which permits simultaneous dual-channel NIR fluorescence imaging. To summarize, ESNFs and MB exhibited fluorescence properties compatible with NIR fluorescence channel \#1 (700 nm) of the FLARE imaging system and NIR fluorescence microscope while ICG and ZW800-3a exhibited fluorescence properties compatible with NIR fluorescence channel \#2 (800 $\mathrm{nm}$ ).
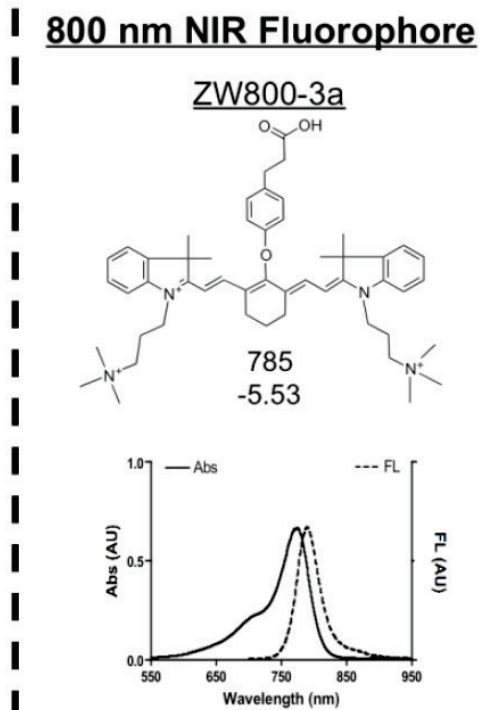

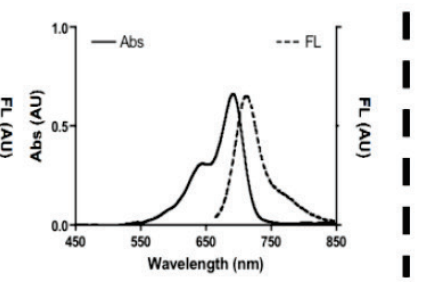


Table I. Optical properties of NIR fluorophores in 100\% FBS supplemented with $50 \mathrm{mM} \mathrm{HEPES,} \mathrm{pH} \mathrm{7.4.}$

\begin{tabular}{|c|c|c|c|c|c|c|}
\hline Property & ESNF11 & ESNF14 & ESNF20 & MB & ZW800-3a & ICG \\
\hline Extinction Coefficient $\left(\varepsilon, \mathrm{M}^{-1} \mathrm{~cm}^{-1}\right)$ & 147,000 & 183,000 & 179,000 & 71,200 & 309,000 & 121,000 \\
\hline Absorbance Maximum (nm) & 656 & 696 & 692 & 665 & 774 & 807 \\
\hline Emission Maximum (nm) & 675 & 718 & 712 & 688 & 789 & 822 \\
\hline Quantum Yield $(\phi, \%)$ & 45.3 & 21.5 & 15.8 & 3.8 & 16.1 & 9.3 \\
\hline Molecular Brightness $\left(\varepsilon \times \phi, \mathrm{M}^{-1} \mathrm{~cm}^{-1}\right)$ & 66,591 & 39,345 & 28,282 & 2,706 & 49,749 & 11,253 \\
\hline
\end{tabular}

A.

A. color Image
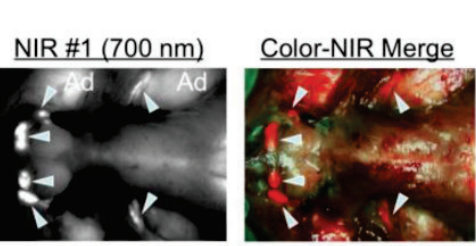

B.


C.

$700 \mathrm{~nm}$ NIR Fluorophores

$800 \mathrm{~nm}$ NIR Fluorophore

D.
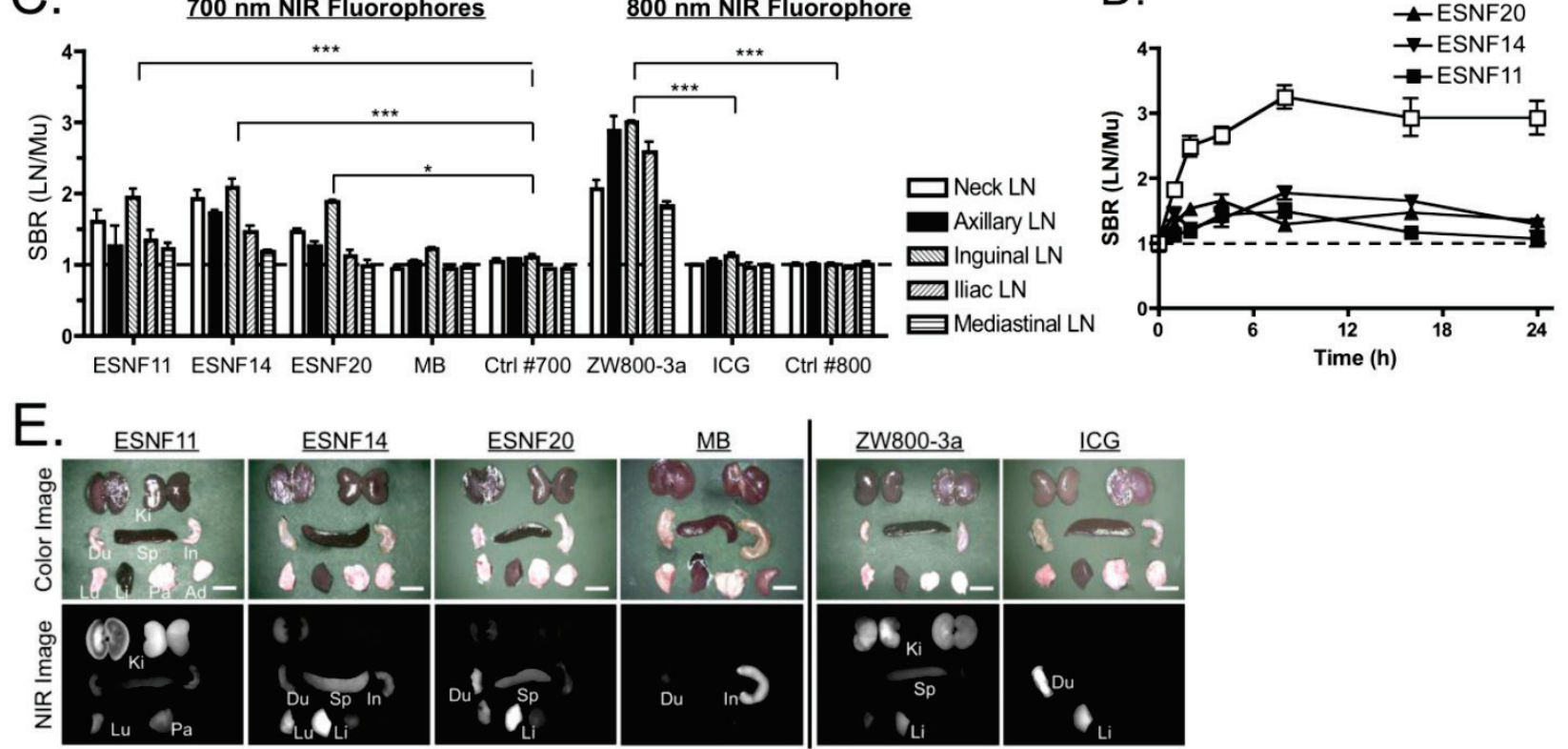

Figure 2. Pan Lymph Node Imaging in Rats: 50 nmol ESNFI4 (A) or 20 nmol ZW800-3a (B) was injected intravenously into SD rats 4 h prior to imaging. In the merged image, pseudo-colored red and green were used for $700 \mathrm{~nm}$ and $800 \mathrm{~nm}$ channels, respectively. LNs are identified with arrowheads. (C) SBR, expressed as the LN to muscle (Mu) ratio (mean \pm SEM) for each agent in all major LN basins 4 h post- injection of 50 nmol ESNF series and MB, or 20 nmol ZW800-3a and ICG. Controls were injected with saline alone. Statistical analysis was performed using one-way ANOVA followed by Tukey's multiple comparison post-test $* P<0.05$, $* * P$ $<0.0$ I, $* * * P<0.00 I$ ). (D) Kinetics of pan lymph node NIR fluorescence. SBR, expressed as the LN to muscle (Mu) ratio (mean \pm SEM) for each agent over time. (E) Biodistribution of intravenously injected NIR fluorophores $4 \mathrm{~h}$ post-injection. Data are from $\mathrm{N}=3$ rats per condition. Ad, white adipose; BI, bladder; Du, duodenum; $\mathrm{He}$, heart; In, intestine; Ki, kidney; Li, liver; Lu, lung; LN, lymph node. Scale bars $=1 \mathrm{~cm}$.

PLN Mapping in Rats. As shown in Figure 2A, ESNF14 accumulated significantly in LNs after a single intravenous injection. However, nonspecific up- take in muscle, adipose, liver, lung, and intestine interfered with clear visualization of PLNs and remained longer than $4 \mathrm{~h}$ post- injection. On the other 
hand, intravenously injected ZW800-3a visualized PLNs with minimum nonspecific tissue uptake, which yielded more than 2-fold higher SBR (LN/Mu) compared to ESNFs. By comparison, there was no significant difference in SBR between $\mathrm{MB}$ and saline controls $4 \mathrm{~h}$ post-intravenous injection (Figure 2C), except for mesenteric LNs, which reabsorb MB from the GI tract after its excretion into bile [20]. In most cases, the SBR (LN/Mu) peaked between $4 \mathrm{~h}$ and $8 \mathrm{~h}$ over the course of 24 hours. Nonspecific background decreased significantly by $24 \mathrm{~h}$ post-injection; however, the signal at the target LNs also diminished rapidly by $8 \mathrm{~h}$ post-injection. There was no significant difference between the SBR (LN/Mu) at $4 \mathrm{~h}$ and later time points up to $24 \mathrm{~h}$ (Figure 2D). Biodistribution of each compound $4 \mathrm{~h}$ post-intravenous injection reveals slow elimination of ESNFs and ZW800-3a compared to MB and ICG.

PLN Mapping in Pigs. Based on the small animal data, we performed PLN imaging in pigs ( 3 animals per fluorophore) at $4 \mathrm{~h}$ post-intravenous injection of each fluorophore. As shown in Figures 3A and B, ZW800-3a showed significantly higher SBR $(\mathrm{LN} / \mathrm{Mu})$ than all of the other fluorophores, which is consistent with the small animal data. ZW800-3a was then injected into 3 pigs and signals compared between PLNs and the varying background tissues (muscle, liver, and kidney) over $4 \mathrm{~h}$. During the distribution phase, namely $\mathrm{T}=0-1 \mathrm{~h}, \mathrm{SBR} \mathrm{LN} / \mathrm{Li}$ and SBR LN/Ki were close to the baseline of 1.0. SBR then gradually increased over $4 \mathrm{~h}$ with peak SBR measured at $4 \mathrm{~h}$ post-intravenous injection (Figure $3 \mathrm{C}$ ).

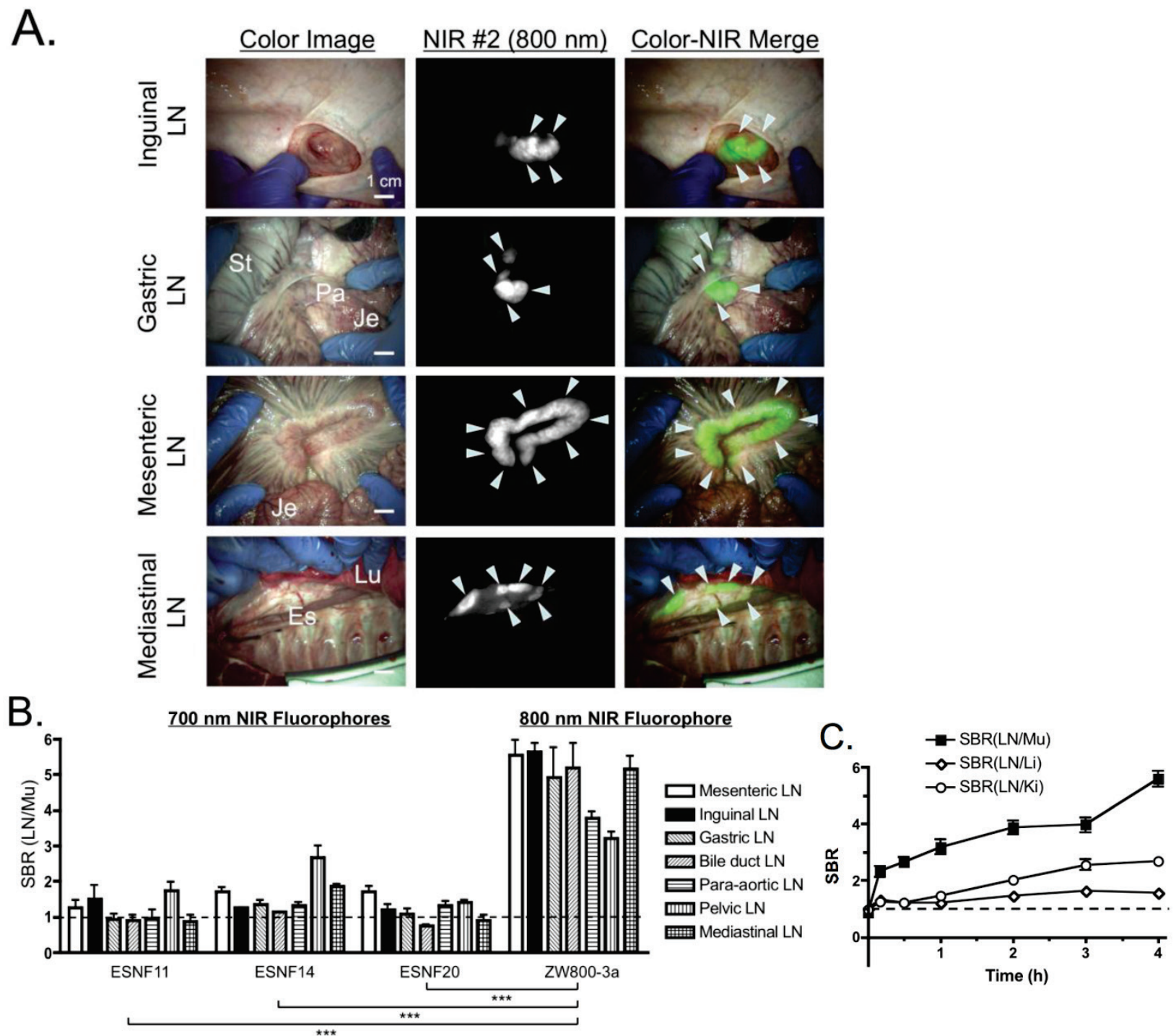

Figure 3. Pan Lymph Node Imaging in Pigs: (A) Inguinal LNs (top), gastric LNs (2nd row), mesenteric LNs (3rd row) and mediastinal LNs (bottom) after intravenous injection of $640 \mathrm{nmol}$ ZW800-3a into $35 \mathrm{~kg}$ Yorkshire pigs $4 \mathrm{~h}$ prior to imaging. Shown are representative images from N = 3 pigs, and pseudo-colored green was used for merged image. LNs are identified with arrowheads. Es, esophagus; Je, jejunum; Lu, lung; LN, lymph node; Pa, pancreas; St, stomach. Scale bars = I cm. (B) SBR, expressed as the LN to muscle (Mu) ratio (mean \pm SEM) for each agent in all major LN basins $4 \mathrm{~h}$ after intravenous injection of I.4 4 mol ESNF series or 640 nmol ZW800-3a. Data are from N = 3 pigs per condition. Statistical analysis of ZW800-3a vs. each ESNF dye was performed using one-way ANOVA followed by Tukey's multiple comparison post-test $(* P<0.05$, $* * P<0.0$ I, $* * * P<0.001)$. (C) Kinetics of pan lymph node NIR fluorescence. SBR, expressed as the LN to muscle (black squares), liver (white diamonds), or kidney (white circles) ratio (mean $\pm \mathrm{SEM}$ ) for each agent over time. $\mathrm{N}=3$ pigs per data point. 
Simultaneous Dual-Channel Imaging of PLN and SLN Mapping in Pigs. Because completion lymphadenectomy is required in a large fraction of SLN biopsies, we attempted to visualize both SLN and PLNs simultaneously by exploiting the dual-NIR channel capability of the FLARE imaging system. Intravenously injected ZW800-3a was selected for 800 $\mathrm{nm}$ channel imaging because low nonspecific background is required for PLN mapping, while locally injected ESNF14 was used for $700 \mathrm{~nm}$ channel imaging of the SLN. Four hours after intravenous injection of $640 \mathrm{nmol} \mathrm{ZW800-3a,} 5 \mathrm{nmol}$ of ESNF14 was injected transvaginally and submucosally into the uterine cer$\operatorname{vix}(\mathrm{N}=3)$. Immediately after injection of ESNF14, the SLN (in this case the common iliac LN) was visualized on FLARE channel \#1. Afterwards, both PLNs (FLARE channel \#2) and the SLN could be imaged simultaneously (Figure 4A).
For histological evaluation, we removed a control LN from the pelvic area before injection of any fluorophore. After injection of both ZW800-3a and ESNF14, we removed a pelvic LN showing signal from only ZW800-3a (i.e., PLN) and the SLN that displayed both ZW800-3a and ESNF14 fluorescence. Expected microscopic LN substructure [35] was confirmed by H\&E staining, with each NIR fluorophore displaying a distinct LN uptake pattern. Control LNs were negative for NIR fluorescence (Figure $4 B$, top). For intravenously injected ZW800-3a, the cortex and supplying vasculature of the LN was bright (Figure 4B, middle). For ESNF14, the subcapsular sinus showed the brightest signal (Figure 4B, bottom), consistent with afferent lymphatic flow from the site of injection.

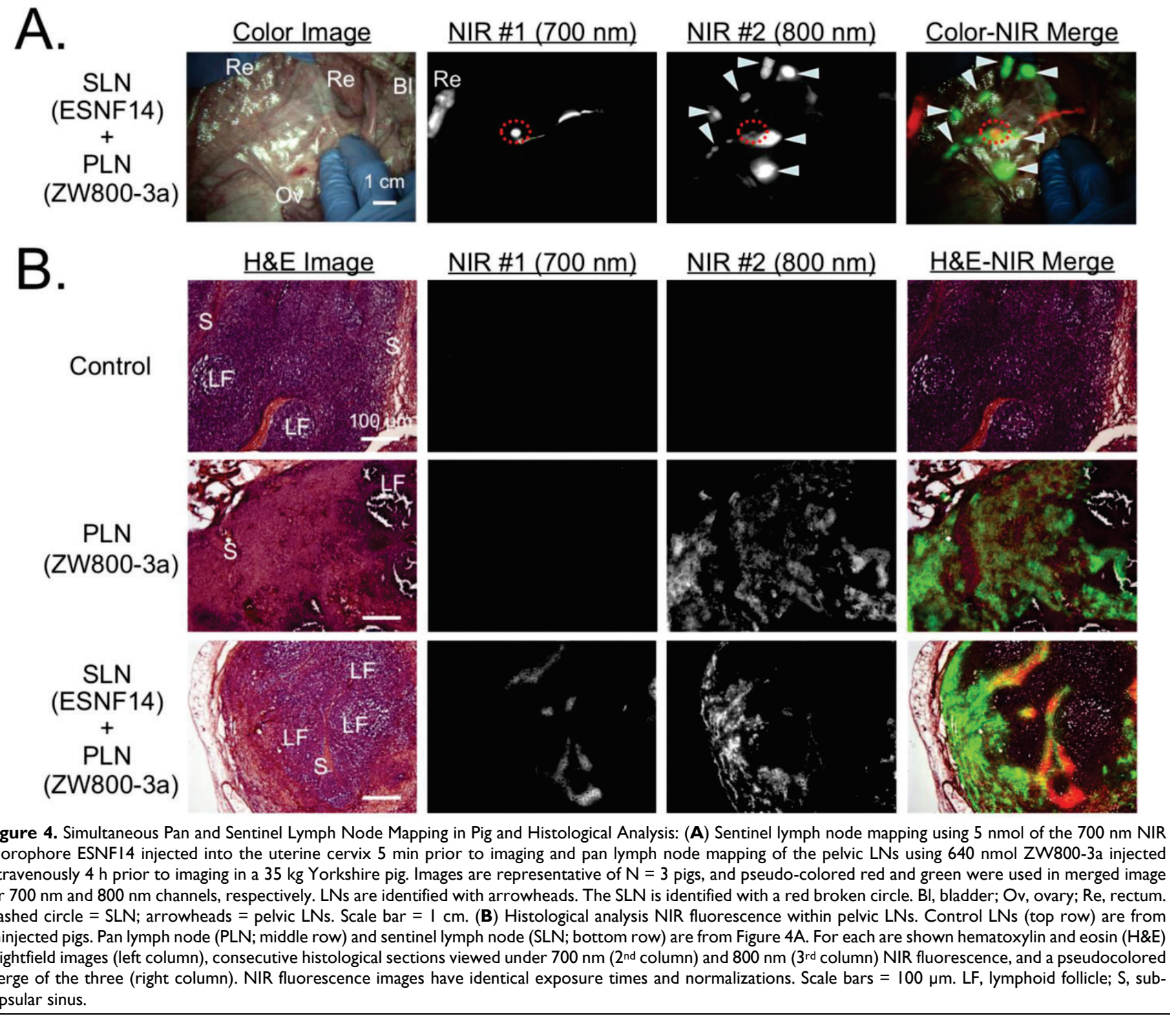




\section{Discussion}

SLN mapping is the standard of care for breast cancer and melanoma, and is also being investigated for the staging of many other solid tumors. In approximately $30 \%$ to $40 \%$ of breast cancer cases $[10,11]$ and $15 \%$ to $20 \%$ of melanoma cases $[12,13]$, though, a positive SLN is found and a completion lymphadenectomy is required. Additionally, the proper staging and treatment of cervical cancer requires an extensive LN dissection in the pelvis and around the descending aorta. Pelvic anatomy is very complicated, and there are currently no methods available to help surgeons to locate all LNs within a basin. As a result, dissection of LNs is often associated with intraoperative morbidity, such as hemorrhage, nerve damage, and long-term morbidity, such as serocele formation and lymphedema [36]. Ideally, a PLN agent could be injected intravenously at a relatively low dose prior to surgery, home specifically to all lymph nodes in the body, and provide real-time, high sensitivity image guidance to the surgeon during lymphadenectomy. Currently available clinical NIR fluorophores, MB and ICG, cannot visualize PLNs after intravenous injection. Josephson et al. used Cy5.5-PGC for NIR imaging of PLN mapping [26]; however, the fluorophore resulted in high nonspecific background uptake in most tissues and organs, especially the liver. A clinically useful agent needs to exhibit low nonspecific uptake in major organs and tissues as well as high signal in LNs [37].

An in vivo pilot study demonstrated that both ESNFs and ZW800-3a are viable SLN tracers, with both visualizing the SLN immediately (within $1 \mathrm{~min}$ ), and with high SBR $(>5.0)$ and long retention time $(>1$ h) after local injection (data not shown). Based on this result, we systematically investigated the possibility of using these agents for mapping all LNs in the body after single intravenous injection. Although all fluorophores displayed reasonably good signal in PLNs, the $700 \mathrm{~nm}$ ESNF series exhibited high nonspecific background uptake in muscle, adipose, kidneys, and liver that limited their utility for PLN mapping. On the other hand, $800 \mathrm{~nm}$ ZW800-3a provided high SBR in PLNs in both small and large animal models as a result of low nonspecific background uptake, higher excitation and emission wavelength (i.e., lower tissue attenuation), lower autofluorescence, and optimal optical properties (Table 1).

It should be noted that the proper statistical assessment of SLN biopsy, which includes sensitivity, specificity, positive predictive value, negative predictive value, and accuracy, is dependent on finding all LNs within a basin. The technology we describe should aid in this endeavor, and might someday permit improved quantitative evaluation of SLN biopsy for cancers other than breast cancer and melanoma.

Although ZW800-3a performs reasonably well as a PLN agent, and has excellent optical properties, its specificity requires additional optimization. The mechanism of uptake in lymph nodes is presently not known, although micro-precipitation is unlikely because of the absence of signal in lung and spleen. The combination of $\log \mathrm{D}$, surface charge, and chemical structure appears to have specificity for LN uptake and retention, with our histological data suggesting that the fluorophore is being delivered via the systemic vasculature. Future studies should be directed at defining the mechanism of uptake and improving specificity, while maintaining current optical properties. cGMP-compatible chemical syntheses will also need to be developed to enable first-in-human clinical trials.

\section{Acknowledgments}

We thank Rita G. Laurence for assistance with animal surgery, Lindsey Gendall and David Burrington, Jr. for editing, and Eugenia Trabucchi for administrative assistance. This study was supported by the following grants: National Institutes of Health grants \#R01-CA-115296 (JVF), \#R01-EB-010022 (JVF and HSC), and \#R01-EB-011523 (HSC and JVF), and a grant from the Dana Foundation (HSC). The views expressed are solely those of the authors and do not necessarily reflect those of the National Institutes of Health.

\section{Conflict of Interest}

FLARE $^{\text {TM }}$ technology is owned by Beth Israel Deaconess Medical Center, a teaching hospital of Harvard Medical School. It has been licensed to the FLARE Foundation, a non-profit organization focused on promoting the dissemination of medical imaging technology for research and clinical use. Dr. Frangioni is the founder and chairman of the FLARE Foundation. The Beth Israel Deaconess Medical Center will receive royalties for sale of FLARE ${ }^{\mathrm{TM}}$ Technology. Dr. Frangioni has elected to surrender post-market royalties to which he would otherwise be entitled as inventor, and has elected to donate pre-market proceeds to the FLARE Foundation. Dr. Frangioni has started three for-profit companies, Curadel, Curadel ResVet Imaging, and Curadel Surgical Innovations, which may someday be non-exclusive sub-licensees of FLARE $^{\mathrm{TM}}$ technology.

\section{References}

1. Morton DL, Wen DR, Wong JH, Economou JS, Cagle LA, Storm FK, et al. Technical details of intraoperative lymphatic mapping for early stage melanoma. Arch Surg. 1992; 127: 392-9. 
2. Nieweg OE, van Rijk MC, Valdes Olmos RA, Hoefnagel CA. Sentinel node biopsy and selective lymph node clearance--impact on regional control and survival in breast cancer and melanoma. European journal of nuclear medicine and molecular imaging. 2005; 32: 631-4.

3. Chen SL, Iddings DM, Scheri RP, Bilchik AJ. Lymphatic mapping and sentinel node analysis: current concepts and applications. CA Cancer J Clin. 2006; 56: 292-309; quiz 16-7.

4. van der Pas MH, Meijer S, Hoekstra OS, Riphagen II, de Vet HC, Knol DL, et al. Sentinel-lymph-node procedure in colon and rectal cancer: a systematic review and meta-analysis. Lancet Oncol. 2011; 12: 540-50.

5. Lips DJ, Schutte HW, van der Linden RL, Dassen AE, Voogd AC, Bosscha K. Sentinel lymph node biopsy to direct treatment in gastric cancer. A systematic review of the literature. Eur J Surg Oncol. 2011; 37: 655-61.

6. Takeuchi H, Kitagawa Y. Sentinel node navigation surgery for esophageal cancer. Gen Thorac Cardiovasc Surg. 2008; 56: 393-6.

7. Balasubramanian SP, Harrison BJ. Systematic review and meta-analysis of sentinel node biopsy in thyroid cancer. Br J Surg. 2011; 98: 334-44.

8. Catarino PA, Goldstraw P. The future in diagnosis and staging of lung cancer: surgical techniques. Respiration. 2006; 73: 717-32.

9. Oonk MH, van de Nieuwenhof HP, de Hullu JA, van der Zee AG. The role of sentinel node biopsy in gynecological cancer: a review. Curr Opin Oncol. 2009; 21: 425-32.

10. Kelley MC, Hansen N, McMasters KM. Lymphatic mapping and sentinel lymphadenectomy for breast cancer. Am J Surg. 2004; 188: 49-61.

11. Kim T, Giuliano AE, Lyman GH. Lymphatic mapping and sentinel lymph node biopsy in early-stage breast carcinoma: a metaanalysis. Cancer. 2006; 106: 4-16.

12. Morton DL, Wen DR, Foshag LJ, Essner R, Cochran A. Intraoperative lymphatic mapping and selective cervical lymphadenectomy for early-stage melanomas of the head and neck. J Clin Oncol. 1993; 11: 1751-6.

13. Elias N, Tanabe KK, Sober AJ, Gadd MA, Mihm MC, Goodspeed B, et al. Is completion lymphadenectomy after a positive sentinel lymph node biopsy for cutaneous melanoma always necessary? . Arch Surg. 2004; 139: 400-4.

14. Kim S, Lim YT, Soltesz EG, De Grand AM, Lee J, Nakayama A, et al. Near-infrared fluorescent type II quantum dots for sentinel lymph node mapping. Nature biotechnology. 2004; 22: 93-7.

15. Tanaka E, Choi HS, Fujii H, Bawendi MG, Frangioni JV. Image-guided oncologic surgery using invisible light: completed pre-clinical development for sentinel lymph node mapping. Ann Surg Oncol. 2006; 13: 1671-81.

16. Frangioni JV. In vivo near-infrared fluorescence imaging. Curr Opin Chem Biol. 2003; 7: 626-34.

17. Ballou B, Ernst LA, Andreko S, Harper T, Fitzpatrick JA, Waggoner AS, et al. Sentinel lymph node imaging using quantum dots in mouse tumor models. Bioconjug Chem. 2007; 18: 389-96.

18. Kim S, Lim C, Na J, Lee Y, Kim K, Choi K, et al. Conjugated polymer nanoparticles for biomedical in vivo imaging. Chem Commun. 2010; 46: $1617-9$.

19. Kobayashi H, Koyama $Y$, Barrett $T$, Hama $Y$, Regino CA, Shin IS, et al Multimodal nanoprobes for radionuclide and five-color near-infrared optical lymphatic imaging. ACS Nano. 2007; 1: 258-64.

20. Troyan SL, Kianzad V, Gibbs-Strauss SL, Gioux S, Matsui A, Oketokoun R, et al. The FLARE intraoperative near-infrared fluorescence imaging system: a first-in-human clinical trial in breast cancer sentinel lymph node mapping. Ann Surg Oncol. 2009; 16: 2943-52.

21. Hutteman M, Choi HS, Mieog JS, van der Vorst JR, Ashitate Y, Kuppen PJ, et al. Clinical translation of ex vivo sentinel lymph node mapping for colorectal cancer using invisible near-infrared fluorescence light. Ann Surg Oncol 2011; 18: 1006-14.

22. Mieog JS, Troyan SL, Hutteman M, Donohoe KJ, van der Vorst JR, Stockdale A, et al. Toward optimization of imaging system and lymphatic tracer for near-infrared fluorescent sentinel lymph node mapping in breast cancer. Ann Surg Oncol. 2011; 18: 2483-91.

23. Schaafsma BE, Mieog JS, Hutteman M, van der Vorst JR, Kuppen PJ, Lowik $\mathrm{CW}$, et al. The clinical use of indocyanine green as a near-infrared fluorescent contrast agent for image-guided oncologic surgery. J Surg Oncol. 2011; 104: 323-32.

24. van der Vorst JR, Hutteman M, Gaarenstroom KN, Peters AA, Mieog JS, Schaafsma BE, et al. Optimization of near-infrared fluorescent sentinel lymph node mapping in cervical cancer patients. Int J Gynecol Cancer. 2011; 21: $1472-8$.

25. Vahrmeijer AL, Hutteman M, van der Vorst JR, van de Velde CJ, Frangioni JV. Image-guided cancer surgery using near-infrared fluorescence. Nat Rev Clin Oncol. 2013; 10: 507-18.

26. Josephson L, Mahmood U, Wunderbaldinger P, Tang Y, Weissleder R. Pan and sentinel lymph node visualization using a near-infrared fluorescent probe. Mol Imaging. 2003; 2: 18-23.

27. Choi HS, Nasr K, Alyabyev S, Feith D, Lee JH, Kim SH, et al. Synthesis and in vivo fate of zwitterionic near-infrared fluorophores. Angew Chem Int Ed Engl. 2011; 50: 6258-63.

28. Choi HS, Ashitate Y, Lee JH, Kim SH, Matsui A, Insin N, et al. Rapid translocation of nanoparticles from the lung airspaces to the body. Nat Biotechnol 2010; 28: 1300-3.

29. Ashitate Y, Vooght CS, Hutteman M, Oketokoun R, Choi HS, Frangioni JV. Simultaneous assessment of luminal integrity and vascular perfusion of the gastrointestinal tract using dual-channel near-infrared fluorescence. Mol imaging. 2012; 11: 301-8

30. Ashitate Y, Stockdale A, Choi HS, Laurence RG, Frangioni JV. Real-time simultaneous near-infrared fluorescence imaging of bile duct and arterial anatomy. J Surg Res. 2012; 176: 7-13.

31. Ashitate Y, Tanaka E, Stockdale A, Choi HS, Frangioni JV. Near-infrared fluorescence imaging of thoracic duct anatomy and function in open surgery and video-assisted thoracic surgery. J Thorac Cardiovasc Surg. 2011; 142: 31-8.

32. Gioux S, Kianzad V, Ciocan R, Gupta S, Oketokoun R, Frangioni JV. High-power, computer-controlled, light-emitting diode-based light sources for fluorescence imaging and image-guided surgery. Mol Imaging. 2009; 8: 156-65.

33. Nakayama A, Bianco AC, Zhang CY, Lowell BB, Frangioni JV. Quantitation of brown adipose tissue perfusion in transgenic mice using near-infrared fluorescence imaging. Mol Imaging. 2003; 2: 37-49.

34. Ashitate Y, Kim SH, Tanaka E, Henary M, Choi HS, Frangioni JV, et al. Two-wavelength near-infrared fluorescence for the quantitation of drug antiplatelet effects in large animal model systems. J Vasc Surg. 2012; 56: 171-80.

35. Willard-Mack CL. Normal structure, function, and histology of lymph nodes. Toxicol Pathol. 2006; 34: 409-24.

36. Beesley V, Janda M, Eakin E, Obermair A, Battistutta D. Lymphedema after gynecological cancer treatment : prevalence, correlates, and supportive care needs. Cancer. 2007; 109: 2607-14.

37. Frangioni JV. The problem is background, not signal. Molecular imaging. 2009; 8 : 303-4. 